headache included fatigue and sleep deprivation (72\%), excitement (65\%), fever $(64 \%)$, sun overexposure (62\%), exercise (49\%), ice cream (23\%), anxiety, chocolate, and carbonated drinks. Children with migraine compared to those with tension headaches had more headaches triggered by ice cream, fear, or anxiety (28-36\% cf $9-13 \%)$, more frequent abdominal pain ( $40 \%$ of $11 \%$ ), they took medication more frequently for pain relief, and were more often absent from day care. Pain-relieving factors, darkened room, vomiting, and medication, were more beneficial in migraine than tension headaches. (Aromaa M, Sillanpaa ML, Rautava P, Helenius H. Childhood headache at school entry. A controlled clinical study. Neurology June 1998;50:1729-1736). (Reprints: Dr Minna Aromaa, Department of Public Health, University of Turku, Lemminkaisenkatu 1, 20520 Turku, Finland).

COMMENT. Palpation of occipital muscles and temporomandibular joints may uncover causes of tension-type headaches in children, leading to effective therapy. Headache triggers are especially frequent in migraine sufferers. Relief may be obtained by sleep and rest $(95 \%)$, darkened room (58\%), vomiting (16\%), eating (29\%), and medication (83\%), most commonly ibuprofen.

\title{
MIGRAINE TWIN STUDIES
}

The influence of genetic versus environmental factors in the etiology of migraine was investigated by studies of two samples of female twin pairs - 154 raised together and 43 raised apart since infancy, in a report from the University of Kansas Medical Center, Kansas City and the University of Minnesota, Minneapolis. Tetrachoric correlations for migraine were higher in monozygotic than in dizygotic twins, for both reared-together and reared-apart samples. The heritability estimate for migraine was $52 \%$. Nonshared environmental factors (accidents, illness, stress) and measurement errors accounted for the remaining variance in liability to migraine. (Ziegler DK, Hur Y-M, Bouchard TJ Jr, Hassanein RS, Barter R. Migraine in twins raised together and apart. Headache June 1998;38:417-422). (Respond: Dr Dewey K Ziegler, Department of Neurology, University of Kansas Medical Center, 3901 Rainbow Blvd, Kansas City, KS 66160).

COMMENT. Genetic factors account for $50 \%$ of migraines in women, and environmental factors such as accidents, illness, and stress are responsible for the remaining variance in liability. These US figures are almost identical to previous studies in Finland and Sweden.

Headache pathogenesis (Welch KMA) and genetics of migraine (Gardner K, Hoffman EP) are reviewed in Current Opinion in Neurology June 1998;11:193-197 and 211-216. Brain excitability is the proposed basis for migraine, and causes of neuronal excitability include mitochondrial defects, disturbed magnesium metabolism, and a calcium channelopathy. Familial hemiplegic migraine has been related to mutations in a brain calcium channel gene residing in chromosome $19 \mathrm{p}$ or chr 1 . The larger group of migraine disorders may be associated with dopamine DRD2 receptor genes.

\section{SEIZURE DISORDERS}

\section{MOZART EFFECT ON SEIZURE ACTIVITY IN THE EEG}

The "Mozart Effect" on epileptiform activity in the EEG of 29 patients, ages 3-47 years, was investigated using brain maps and computerized analyses at the University of Illinois Medical Center, Chicago, IL. The Sonata for Two Pianos in D Major (K.448) was selected as in previous cognitive and EEG studies, and the 